\title{
Incorporating evanescent modes and flow losses into reference impedances in acoustic Thévenin calibration
}

Nørgaard, Kren Rahbek; Fernandez Grande, Efren

Published in:

Journal of the Acoustical Society of America

Link to article, DOI:

$10.1121 / 1.5010891$

Publication date:

2017

Document Version

Publisher's PDF, also known as Version of record

Link back to DTU Orbit

Citation (APA):

Nørgaard, K. R., \& Fernandez Grande, E. (2017). Incorporating evanescent modes and flow losses into reference impedances in acoustic Thévenin calibration. Journal of the Acoustical Society of America, 142(5), 3013-3024. https://doi.org/10.1121/1.5010891

\section{General rights}

Copyright and moral rights for the publications made accessible in the public portal are retained by the authors and/or other copyright owners and it is a condition of accessing publications that users recognise and abide by the legal requirements associated with these rights.

- Users may download and print one copy of any publication from the public portal for the purpose of private study or research.

- You may not further distribute the material or use it for any profit-making activity or commercial gain

- You may freely distribute the URL identifying the publication in the public portal 
Incorporating evanescent modes and flow losses into reference impedances in acoustic Thévenin calibration

Kren Rahbek Nørgaard, Efren Fernandez-Grande, and Søren Laugesen

Citation: The Journal of the Acoustical Society of America 142, 3013 (2017);

View online: https://doi.org/10.1121/1.5010891

View Table of Contents: http://asa.scitation.org/toc/jas/142/5

Published by the Acoustical Society of America 


\title{
Incorporating evanescent modes and flow losses into reference impedances in acoustic Thévenin calibration
}

\author{
Kren Rahbek Nørgaard ${ }^{\text {a) }}$ and Efren Fernandez-Grande \\ Acoustic Technology, Department of Electrical Engineering, Technical University of Denmark, Ørsteds Plads, \\ Building 352, Kongens Lyngby, DK-2800, Denmark
}

\author{
Søren Laugesen \\ Interacoustics Research Unit, Technical University of Denmark, Ørsteds Plads, Building 352, Kongens \\ Lyngby, DK-2800, Denmark
}

(Received 5 May 2017; revised 25 October 2017; accepted 27 October 2017; published online 16 November 2017)

This paper proposes an alternative approach to acoustic Thévenin calibration of an ear probe. An existing methodology derives the Thévenin-equivalent source parameters from the measured probe pressures in a number of short waveguides by solving an overdetermined system of equations. This existing methodology is affected by errors caused by evanescent modes when the waveguide model lengths are estimated. These errors introduce a parallel acoustic compliance into the source parameters. The proposed methodology takes into account evanescent modes and flow losses in the transition between the probe tube and waveguides during calibration. This is achieved by positioning the probe tube, without an ear tip, flush with the input plane in waveguides of well-defined dimensions and utilizing the physical rather than estimated lengths to calculate the analytical waveguide models. Terms that model evanescent modes and flow losses are added to the plane-wave impedance and adjusted to minimize the calibration error. It is shown that this method can reduce the calibration error across a wide frequency range and remove the parallel compliance from the source parameters. This approach leads to an independence of the source parameters on the calibration waveguide radius, though subsequent impedance measurements are still affected by evanescent modes. (ㅇ 2017 Acoustical Society of America. https://doi.org/10.1121/1.5010891

[CAS]

Pages: $3013-3024$

\section{INTRODUCTION}

The measurement of acoustic impedance in the ear canal across a wide range of frequencies has received increasing attention in recent years. Many studies have found clinically relevant information in the ear-canal reflectance (Piskorski et al., 1999; Keefe et al., 2000; Feeney and Keefe, 2001; Keefe et al., 2012; Ellison et al., 2012; Merchant et al., 2014), and more recently, ear-canal impedance and reflectance have found application in estimating hearing thresholds (Withnell et al., 2009; Souza et al., 2014). In addition, they can be used for adjusting stimulus levels in otoacoustic emission measurements (Scheperle et al., 2008; McCreery et al., 2009; Lewis et al., 2009; Scheperle et al., 2011), and have shown potential in estimating the emitted pressure level from the ear (Charaziak and Shera, 2017). A few commercial systems also exist that utilize this type of impedance measurement, e.g., the Titan (Interacoustics A/S, Middelfart, Denmark) and the HearID (Mimosa Acoustics, Inc., Champaign, IL). To facilitate the measurement of acoustic impedance of the ear canal or a waveguide, the ear probe must be characterized by its source parameters in a preceding calibration procedure. The source parameters completely describe the linear response and impedance characteristics of the probe. A few different calibration methods exist, which

\footnotetext{
a) Also at: Interacoustics A/S, Audiometer Allé 1, DK-5500 Middelfart, Denmark. Electronic mail: krng@interacoustics.com
}

are all affected by acoustic phenomena related either to variations in calibration hardware or probes. If not properly accounted for, these phenomena inherently introduce errors into the source parameters and thereby into subsequent impedance measurements. Thus, the comparison of data obtained using different measurement systems may be limited if these systems are affected differently by such phenomena.

The acoustic source parameters of an ear probe can be characterized by a Thévenin-equivalent model, the source pressure and source impedance, as described by Allen (1985), Keefe et al. (1992), and Voss and Allen (1994). This is achieved by presenting the probe to a number of different acoustic loads, typically hard-walled, rigidly terminated waveguides, for which the analytical plane-wave input impedances can be precisely calculated using a transmission-line model (Keefe, 1984). The probe pressures in each load are measured and the source parameters are obtained by solving an overdetermined system of equations using the leastsquares method. The probe is inserted into each waveguide using an ear tip and so the physical lengths of the waveguides are not known due to the uncertainty associated with the insertion. The lengths used to calculate the analytical impedances of each waveguide are therefore estimated. This multi-tube calibration methodology will be referred to here as the existing calibration. The errors introduced into the source parameters in the existing methodology of this multitube calibration depend on evanescent modes in the area 
discontinuity between the probe tube and calibration waveguides. These errors especially become apparent in measurements of waveguides with radii different from the calibration waveguides. The comparison of data obtained with different probe systems can therefore be limited since the contribution from evanescent modes depends on the configuration of the probe-tube apertures.

This paper proposes a calibration methodology that models the behavior of evanescent modes and flow losses during calibration that result from the geometrical mismatch between the probe tube and waveguide. A set of calibration hardware is used that can be manufactured with high precision for increased repeatability in obtaining the source parameters. In this way, errors introduced into subsequent impedance measurements are independent of the ratio of calibration to measurement waveguide radii, but will still include evanescent modes and other effects that might affect it. The proposed methodology could therefore provide a step forward in increasing the validity of ear-canal impedance and reflectance measurements, estimating input levels to the ear, and measuring otoacoustic emissions.

\section{EXISTING METHODS AND EVANESCENT MODES}

The overdetermined system of equations describing the linear relation between the unknown source parameters (the source pressure $P_{\mathrm{s}}$ and source impedance $Z_{\mathrm{s}}$ ), the measured probe pressures $P_{i}$, and modeled reference impedances $Z_{i}$ in the acoustic Thévenin-equivalent model is given from the existing literature (e.g., Allen, 1985),

$$
\left[\begin{array}{cc}
Z_{1} & -P_{1} \\
Z_{2} & -P_{2} \\
\vdots & \vdots \\
Z_{N} & -P_{N}
\end{array}\right]\left[\begin{array}{c}
P_{\mathrm{s}} \\
Z_{\mathrm{s}}
\end{array}\right]=\left[\begin{array}{c}
P_{1} Z_{1} \\
P_{2} Z_{2} \\
\vdots \\
P_{N} Z_{N}
\end{array}\right]
$$

for $N$ waveguides. The source parameters are fitted using a least-squares approach by minimizing the cost function,

$$
\epsilon_{\mathrm{ls}}=\sum_{i=1}^{N}\left|Z_{i} P_{\mathrm{s}}-P_{i} Z_{\mathrm{s}}-P_{i} Z_{i}\right|^{2}
$$

Solving for the source parameters in the normal equations yields

$$
\left[\begin{array}{c}
P_{\mathrm{s}} \\
Z_{\mathrm{s}}
\end{array}\right]=\frac{1}{\Delta}\left[\begin{array}{cc}
\sum_{i=1}^{N}\left|P_{i}\right|^{2} & \sum_{i=1}^{N} P_{i} Z_{i}^{*} \\
\sum_{i=1}^{N} P_{i}^{*} Z_{i} & \sum_{i=1}^{N}\left|Z_{i}\right|^{2}
\end{array}\right]\left[\begin{array}{c}
\left.\sum_{i=1}^{N} P_{i} Z_{i}\right|^{2} \\
-\sum_{i=1}^{N}\left|P_{i}\right|^{2} Z_{i}
\end{array}\right],
$$

with

$$
\Delta=\sum_{i=1}^{N}\left|P_{i}\right|^{2} \sum_{i=1}^{N}\left|Z_{i}\right|^{2}-\left|\sum_{i=1}^{N} P_{i} Z_{i}^{*}\right|^{2} .
$$

Previous studies have estimated the waveguide model lengths by iteratively adjusting them and calculating the source parameters from Eq. (3), choosing the lengths that provide the lowest calibration error. The calibration error is a quantity different from the least-squares cost function [Eq. (2)]. It has typically been defined as the ability of the source parameters, obtained in each iteration of waveguide lengths using Eq. (3), to estimate the reference impedances, probe pressures, or a similar quantity, averaged across some frequency range. Given the formulation of the linear system in Eq. (1), the least-squares cost function [Eq. (2)] weighs impedance maxima more heavily in calculating the best-fit source parameters. In this way, the source parameters always align to provide a low calibration error in impedance maxima. Any mismatches in waveguide model lengths relative to the measured probe pressures will thus mainly be represented in the calibration error near impedance minima, due to their low weight in determining the source parameters. With this methodology, the placement in frequency of the probe-pressure minima therefore determines these lengths used to derive the source parameters. Authors have not always been consistent in specifying exactly which error is minimized during the length-optimization procedure and across which frequency range. It is thus not possible to generalize this assumption to apply to all existing publications on the multi-tube calibration method.

Evanescent modes appear in geometrical discontinuities between two waveguides in addition to the plane-wave, propagating mode, as higher-order, non-propagating modes are excited. However, these evanescent modes decay exponentially from the discontinuity along the waveguide axis. Keefe et al. (1992) and Voss and Allen (1994) protruded the microphone tube beyond the speaker tube in order to eliminate the contribution from evanescent modes to the measured probe pressure. However, this approach includes the excess piece of waveguide between the speaker and microphone tubes in the source parameters. Thus, errors are introduced into subsequent measurements in waveguides of radii different from the calibration waveguides (Huang et al., 2000), since the characteristic impedance $Z_{0}$ of this piece of waveguide changes with waveguides radius. Siegel and Neely (2017) physically modified the tip of the probe tube of an ER-10X ear probe (Etymōtic Research, Inc., Elk Grove Village, IL) and thereby reduced the effect of evanescent modes on the calibration. They verified the effect in terms of the visually inspected causality of the source impedance in the time domain, compared to a calibration obtained with an unmodified ear probe tube. However, this probe-tube modification resembles the protrusion of the microphone tube beyond the speaker tube as utilized by Keefe et al. (1992) and Voss and Allen (1994). The approach relies on the shape of the small flange, typically present in rubber ear tips, retaining its shape. It is possible that this flange could vary with the tightness of the fit in an ear canal, though the potential associated errors have not been investigated.

Probes with flush speaker and microphone tubes are thus preferred, although the measured probe pressures are consequently affected by evanescent modes. Evanescent modes can be approximated by the addition of the impedance of an acoustic inertance $L$ to the acoustic, plane-wave impedance $Z_{\mathrm{pw}}$ (Keefe and Benade, 1981; Fletcher et al., 
2005). Thus, the load impedance that the probe is subject to $Z_{\text {load }}$, affected by evanescent modes, can be approximated as

$$
Z_{\mathrm{load}} \simeq Z_{\mathrm{pw}}+j \omega L,
$$

where $j$ is the unit imaginary number and $\omega$ is the angular frequency. This has the effect of translating the position of impedance minima in frequency, proportional to $L / Z_{0}$, since it effectively changes the ordinate zero-crossings of the imaginary part of the impedance. Through the assumed length-optimization procedure, the waveguide model lengths are indirectly determined from probe-pressure minima that are already affected by evanescent modes. These probepressure minima are translated in frequency in the same way as the impedance minima, and the estimated lengths are consequently physically incorrect. By applying a plane-wave impedance model to derive the analytical impedances of the waveguides, a translation of maxima in the reference impedances relative to the true waveguide impedances is achieved. This occurs as impedance maxima appear halfway between minima in the plane-wave model. Due to the heavier weight of impedance maxima in the least-squares cost function [Eq. (2)] and the lengths estimated through the error-optimization routine, this effect of translated impedance maxima is inherited into the source parameters.

In practice, the evanescent-modes term is close to proportional to frequency. Accordingly, the referenceimpedance maxima will also be translated proportionally to frequency in the same direction, relative to the true waveguide impedance maxima. This effect of translated impedance maxima is physically similar to adding the impedance of a parallel acoustic compliance $C$ to the load impedance $Z_{\text {load }}$, affected by evanescent modes. The measured impedance $Z_{\text {meas }}$, as obtained using the existing methodology, can thus be estimated

$$
\begin{aligned}
& Z_{\text {meas }} \simeq\left(\frac{1}{Z_{\text {load }}}+j \omega C\right)^{-1}, \\
& Y_{\text {meas }} \simeq Y_{\text {load }}+j \omega C,
\end{aligned}
$$

where $Y$ denotes admittance. Similar to a series inertance, this compliance effectively changes the ordinate zerocrossings of the imaginary part of admittance and thus translates impedance maxima in frequency. The compliance is phantom in the sense that it is not the result of the physical presence of any parallel cavity to the acoustic load during calibration, but still appears as a parallel component in subsequent measurements. The size of the parallel compliance is defined by the evanescent-modes inertance $L$ during calibration and remains constant thereafter, though the translation of impedance maxima is proportional to $C Z_{0}$. Conversely, the evanescent-modes inertance depends on the configuration of the individual probe-tube apertures and the ratio of their dimensions to the radius of the waveguide. In waveguides of radii identical to the calibration waveguides, the measured impedance appears as one consisting solely of plane waves since impedance minima and maxima are translated equivalently. However, measurements in waveguides

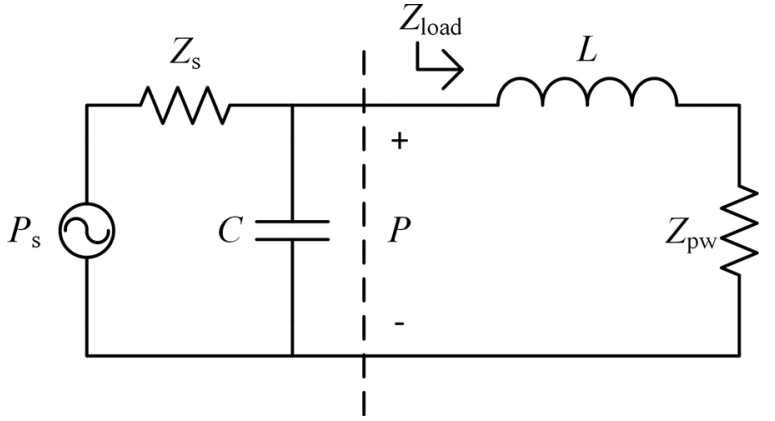

FIG. 1. Thévenin-equivalent circuit diagram of the ear probe inserted into an acoustic load, including the source parameters (the source pressure $P_{\mathrm{s}}$ and source impedance $Z_{\mathrm{s}}$ ), ideal, plane-wave load impedance $Z_{\mathrm{pw}}$, load impedance affected by evanescent modes $Z_{\text {load }}$, and probe pressure $P$ of a waveguide. The inductor $L$ represents the evanescent-modes inertance. The dotted line indicates the transition between the probe on the left-hand side and the acoustic load on the right-hand side. The capacitor $C$ represents the phantom parallel compliance affecting subsequent measurements when waveguide lengths are estimated in the presence of evanescent modes during calibration.

of radii different from the calibration waveguides are affected by errors as impedance minima and maxima are now translated differently. The effect is more profound in waveguides with radii smaller than the calibration waveguides, due to the increased characteristic impedance. From the relationship between the translation of impedance extrema with $L, C$, and $Z_{0}$, this parallel compliance can be estimated

$$
C \simeq \frac{L}{Z_{0}^{2}},
$$

where $L$ and $Z_{0}$ are the quantities present during calibration. The Thévenin-equivalent circuit diagram of the probe and an acoustic load, including the series evanescent-modes inertance $L$ and parallel compliance $C$, is presented in Fig. 1 .

\section{METHODS}

\section{A. Proposed calibration methodology}

An alternative calibration methodology is proposed that is based on the existing multi-tube calibration methodology. However, the tip of the probe tube is placed, without an ear tip, exactly in the input plane of uniform waveguides of well-defined lengths and radii. The measured probe pressures $P_{i}$ in each calibration waveguide essentially represent the averaged sound pressures on the microphone probe-tube aperture in the input plane. In response to a volume flow $U_{i}$ from the speaker probe-tube aperture in the input plane, the ratio of these quantities yields what will be referred to as the transfer impedance between the speaker and microphone apertures. These are the true acoustic impedances $Z_{i, \text { true }}$ that the probe is subject to during calibration

$$
Z_{i, \text { true }}=\frac{P_{i}}{U_{i}}
$$

According to the Thévenin-equivalent model, a measured probe pressure $P_{i}$ is the result of a voltage division of the 
source pressure $P_{\mathrm{s}}$ between the source impedance $Z_{\mathrm{s}}$ and this transfer impedance $Z_{i \text {,true }}$. Thus, this transfer impedance will be modeled rather than the plane-wave impedance. In this way, subsequent impedance measurements will also yield this transfer impedance, including evanescent modes and other effects that might affect it, such as the ear tip. However, the utilization of physical waveguide lengths ensures that impedance maxima are correctly positioned, and the parallel compliance should thus be eliminated from the source parameters.

The first step of the calibration procedure is to acquire the probe pressures $P_{i}$ from the probe inserted into each of the waveguides. Afterward, an initial calibration can be derived from Eq. (3) by calculating the reference impedances $Z_{i}$ from a transmission-line model using the known, physical lengths of the waveguides $l_{i}$,

$$
Z_{i, \mathrm{pw}}=Z_{0} \operatorname{coth} \Gamma l_{i},
$$

and setting $Z_{i}=Z_{i, \mathrm{pw}}$. The characteristic impedance $Z_{0}$ and propagation constant $\Gamma$ are calculated from Keefe (1984).

The next step in the proposed methodology is to include the effect of evanescent modes in the reference impedances used to calculate the source parameters. For each waveguide, an acoustic inertance $L_{i}$ is added to the analytical planewave impedance such that the reference impedances of each waveguide become

$$
Z_{i}=Z_{i, \mathrm{pw}}+j \omega L_{i}
$$

The size of the inertance for each waveguide is iteratively adjusted such that the relative calibration error for the associated waveguide,

$$
\epsilon_{i}=\left|1-\frac{\hat{Z}_{i}}{Z_{i}}\right|,
$$

is minimized in a region, averaged around the first probepressure minimum. Here, $\hat{Z}_{i}$ are the estimated impedances of each waveguide, calculated from the derived source parameters $P_{\mathrm{s}}$ and $Z_{\mathrm{s}}$ in each iteration of $L_{i}$, and measured probe pressures $P_{i}$,

$$
\hat{Z}_{i}=Z_{\mathrm{s}} \frac{P_{i}}{P_{\mathrm{s}}-P_{i}} .
$$

A minimization of the relative error in the imaginary part of the impedances, $\epsilon_{i, \operatorname{Im}}=\left|1-\operatorname{Im} \hat{Z}_{i} / \operatorname{Im} Z_{i}\right|$, and an initial value for the evanescent-modes inertances $L_{i}$, obtained from a previous calibration, adds a level of robustness to the method. Ideally, identical evanescent-modes inertances could be applied for each waveguide if they have the same radius. However, mechanical tolerances inevitably cause small variations in the probe insertion for each waveguide, and the inertance is allowed to differ between waveguides. If an accurate calibration toward high frequencies is desired or several impedance minima are contained within the considered frequency range, it may be necessary to adopt a small addition to the imaginary term

$$
Z_{i}=Z_{i, \mathrm{pw}}+j \omega\left(L_{i}+\omega^{2} L_{i}^{\prime}\right)
$$

The purpose of this factor $L_{i}^{\prime}$ is to model the slight deviation of the evanescent-modes inertance from being proportional to frequency (Keefe and Benade, 1981; Fletcher et al., 2005).

Evanescent modes are by far the largest source of discrepancy between the plane-wave impedance and the transfer impedance between the speaker and microphone apertures (Fletcher et al., 2005). However, fluid motion tangential to the input plane introduces additional variation in the apparent damping of impedance minima. Fletcher et al. (2005) used a term proportional to the square root of frequency, scaled by a loss factor, to compensate for this phenomenon since it approximates a linear, acoustic loss model. As the acoustic flow transitions from the narrow speaker tube in a probe to a waveguide, a spreading flow is induced in proximity to this transition with a large velocity gradient toward the edge at the end of this tube. Such a velocity gradient has to be balanced by an equivalent pressure drop due to conservation of momentum, causing an apparent lower pressure on the probe microphone than predicted by the plane-wave impedance model. This is incorporated into the reference impedances using the same model as utilized by Fletcher et al. (2005) and the loss factors $\zeta_{i}$,

$$
Z_{i}=Z_{i, \mathrm{pw}}+j \omega\left(L_{i}+\omega^{2} L_{i}^{\prime}\right)+\sqrt{\omega} \zeta_{i} .
$$

Similar to the evanescent-modes inertance, the loss factors are adjusted iteratively for each waveguide to provide the lowest-possible, relative calibration error [Eq. (12)] averaged around the first impedance minimum for each waveguide. Due to the nature of evanescent modes in translating impedance minima, this compensation for flow losses cannot be accomplished until the calibration is unaffected by errors due to evanescent modes. Additionally, the relative error in the real part of the impedance, $\epsilon_{i, \operatorname{Re}}=\left|1-\operatorname{Re} \hat{Z}_{i} / \operatorname{Re} Z_{i}\right|$, can be utilized and an initial value supplied for the loss factors for the specific probe and waveguide for increased robustness. The loss factors are also allowed to differ between waveguides since slight offsets of the probe relative to the input plane would cause a difference in the coupling of the acoustic flow to the waveguide.

\section{B. Equipment and measurements}

The measurements reported in this study were carried out using an RME FireFace UC sound card (RME Audio, Haimhausen, Germany), controlled through custom-written MATLAB (The MathWorks, Inc., Natick, MA) software and the third-party utility playrec. ${ }^{1}$ A Titan-based ear probe (Interacoustics A/S, Middelfart, Denmark) was used, but modified to improve the high-frequency performance and reduce internal cross talk.

Probe pressures were obtained by applying a frequencyequalized, wideband chirp to the probe as to provide a flat probe-pressure in a non-reflecting load of radius similar to an adult ear canal. The chirp was played back in the probe in 128 phase-locked, 2048-sample blocks at a sampling rate of 
$44.1 \mathrm{kHz}$, which were each recorded using the probe microphone and averaged to reduce the noise in the measurements.

The waveguides constructed for the presented results, utilizing the proposed calibration methodology, were of lengths $l_{i}=1.2,1.45,1.75$, and $2 \mathrm{~cm}$, all with a radius of $a=2 \mathrm{~mm}$. A cross-sectional view of the insertion of a probe into a waveguide, sketching the construction principle, is shown in Fig. 2. The waveguide lengths were chosen, as far as possible, to avoid overlapping impedance extrema (Scheperle et al., 2011). The radius was chosen to minimize the influence of potential cross talk in the probe, expose the smallest possible part of the rubber section to the acoustic domain, and ensure that evanescent modes have decayed sufficiently before being reflected at the waveguide termination. Also, a small radius should reduce the size of a potential parallel compliance inherited into the source parameters as a result of tolerances in lengths.

In addition, a set of brass calibration waveguides ${ }^{2}$ were used to calibrate the probe using the existing methodology of estimating waveguide lengths as a benchmark. The lengths of each waveguide were estimated by minimizing the calibration error, averaged around the first impedance minimum. The

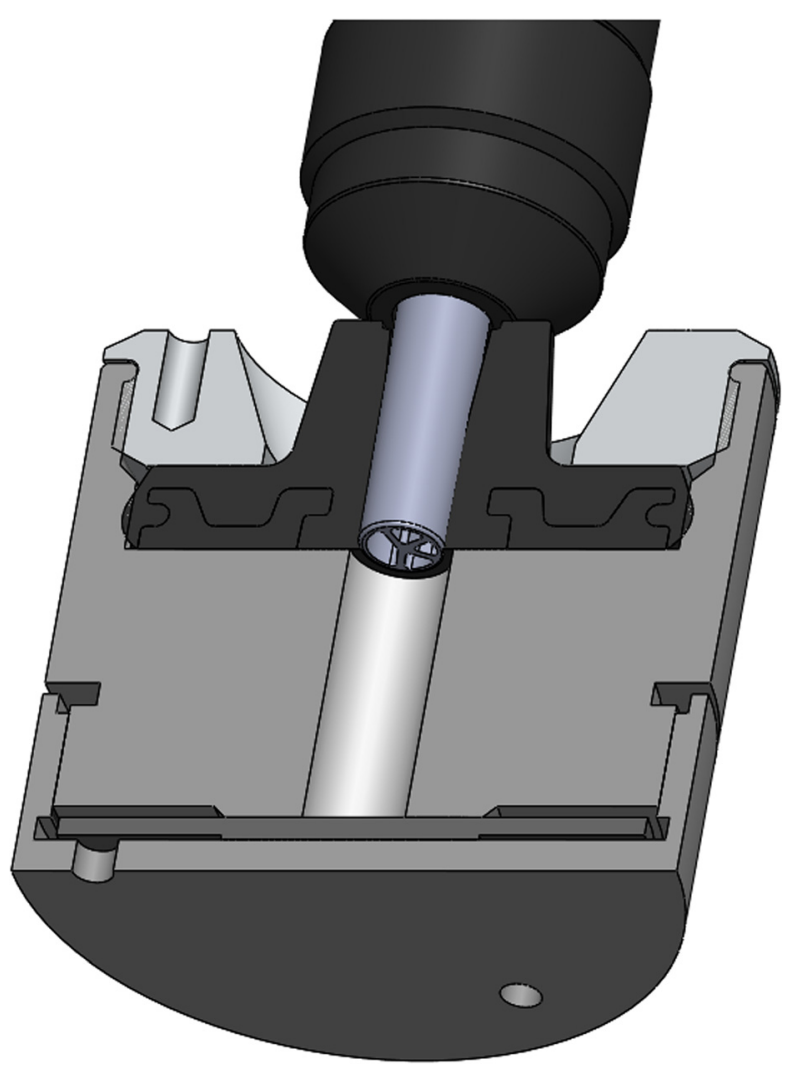

FIG. 2. (Color online) Cross-sectional view of a calibration waveguide utilized for the proposed calibration methodology with the ear probe inserted into the input plane, sketching the construction principle. The workpieces are machined in stainless steel and the actual waveguides are drilled, resulting in a precise, consistent, and smooth bore. The specified tolerances in length and diameter for the workpieces are $\pm 0.01 \mathrm{~mm}$. The probe is held in place by a hard rubber part that aligns with the outer shape and length of the probe tube toward the probe body and ensures a sealed, precise, and flush positioning of the tip of the probe tube in the reference input plane of the waveguides. The waveguides are terminated by a polyoxymethylene disk that is pressed onto the end of the workpieces. waveguides were constructed similar to McCreery et al. (2009), though without the coupler, i.e., the probe was manually removed and reinserted. These brass waveguides were of lengths $l_{i}=1.4,1.7,2$, and $2.3 \mathrm{~cm}$ and radius $a=4 \mathrm{~mm}$. The probe was inserted into the brass waveguides using a standard, mushroom-shaped, green, $9 \mathrm{~mm}$ rubber ear tip (Sanibel Supply, Middelfart, Denmark), and the specified lengths do not necessarily represent the actual lengths from the probe tip.

Two evaluation waveguides, both of lengths $l=1.8 \mathrm{~cm}$, were used for evaluating and comparing the proposed methodology and the considered realization of the existing methodology:

(1) A brass waveguide of radius $a=4 \mathrm{~mm}$, inserting the probe using the ear tip.

(2) A waveguide of radius $a=3.15 \mathrm{~mm}$, using the same construction principle as the proposed calibration waveguides in Fig. 2, i.e., inserting the probe without an ear tip.

\section{SIMULATIONS}

\section{A. Methodology}

A simulation was set up to examine the effect of estimating the waveguide lengths in the presence of evanescent modes given the considered realization of the existing calibration methodology. The probe pressures $P_{i}$ in a set of calibration waveguides were simulated using the voltage division of an arbitrary source pressure $P_{\mathrm{s}}$ between a given source impedance $Z_{\mathrm{s}}$ and simulated waveguide impedances. For the simulation to be comparable with actual measurements, it is important that an appropriate source impedance is chosen, since this parameter determines the relative difference in probe pressures for different waveguides, and thereby affects the weighting of errors in the least-squares cost function [Eq. (2)]. Thus, the source impedance as obtained from the proposed calibration method was used, which is presented later. The simulated, plane-wave impedances included the effects of evanescent modes, modeled by the addition of an acoustic inertance $L$ as in Eq. (11),

$$
P_{i}=P_{\mathrm{s}} \frac{Z_{i, \mathrm{pw}}+j \omega L}{Z_{\mathrm{s}}+Z_{i, \mathrm{pw}}+j \omega L} .
$$

Based on these analytically calculated probe pressures, affected by evanescent modes, the calibration was carried out by adjusting the waveguide lengths such that the relative calibration error [Eq. (12)] was minimized in the region averaged around the first impedance minimum and thus aligning reference-impedance minima with probe-pressure minima. A new set of biased source parameters $P_{\mathrm{s}}^{\prime}$ and $Z_{\mathrm{s}}^{\prime}$ were then obtained, inherently different from those given as input to the simulation due to the parallel compliance. These biased source parameters were compared to the correct source parameters $P_{\mathrm{s}}$ and $Z_{\mathrm{s}}$ and used to investigate the effect on measuring the impedance in waveguides different from the calibration waveguides. This was done by first determining the probe pressure $P$ in an evaluation waveguide using a simulated impedance $Z_{\mathrm{pw}}$, a representative evanescent-modes inertance $L$, and the true source parameters $P_{\mathrm{s}}$ and $Z_{\mathrm{s}}$, similar to Eq. (16), 


$$
P=P_{\mathrm{s}} \frac{Z_{\mathrm{pw}}+j \omega L}{Z_{\mathrm{s}}+Z_{\mathrm{pw}}+j \omega L} .
$$

Based on this probe pressure, the estimated impedances were now calculated using the given, correct, and obtained biased source parameters

$$
\begin{aligned}
& Z=Z_{\mathrm{s}} \frac{P}{P_{\mathrm{s}}-P}=Z_{\mathrm{pw}}+j \omega L, \\
& Z^{\prime}=Z_{\mathrm{s}}^{\prime} \frac{P}{P^{\prime}{ }_{\mathrm{s}}-P} .
\end{aligned}
$$

The impedance measured using the correct source parameters in Eq. (18) simply yields the simulated impedance. The corresponding reflectances $R_{\mathrm{pw}}, R$, and $R^{\prime}$ were calculated from the impedances using the known characteristic impedance $Z_{0}$ of a waveguide. To assess the similarity of the effect on the biased source parameters with a parallel compliance, such negative compliance was added in parallel with the correct source impedance,

$$
Z_{\mathrm{s}} \| C=\left(\frac{1}{Z_{\mathrm{s}}}-j \omega C\right)^{-1}
$$

The compliance changes its sign of operation when it is inherited into the source parameters as it is not physically present during calibration, but rather unintentionally modeled in the reference impedances. The size of the compliance was estimated from Eq. (8).

The evanescent-modes inertances $L$ for the calibration and evaluation waveguides were obtained analytically for the geometry of the probe tube utilized in this study and a specified waveguide radius $a$. This was done using an approach similar to Fletcher et al. (2005), who derived the evanescent-modes inertance for the axisymmetric case of a circular line source and point microphone, extended into a three-dimensional, cylindrical coordinate system. From the radial $r$ and azimuthal $\phi$ coordinates, the boundary condition for the volume-flow injecting face in the input plane was defined as an annular sector of the circular input plane, $a_{1}<r<a_{2}$ and $\phi_{a_{1}}<\phi<\phi_{a_{2}}$, representing the probespeaker aperture. Sound pressure was averaged across another annular sector, $b_{1}<r<b_{2}$ and $\phi_{b_{1}}<\phi<\phi_{b_{2}}$, representing the probe-microphone aperture. Similar to Eq. (15) in Fletcher et al. (2005), the evanescent-modes contribution to the plane-wave impedance $Z_{\mathrm{pw}}$ can be calculated from

$$
\begin{aligned}
Z_{\text {load }}= & Z_{\mathrm{pw}}+\sum_{m=0}^{\infty} \sum_{n=0}^{\infty} \frac{2 \omega \rho\left(1-\delta_{m 0} \delta_{0 n}\right)}{k_{m n} M_{m n}\left(a_{2}^{2}-a_{1}^{2}\right)\left(\phi_{a_{2}}-\phi_{a_{1}}\right)\left(b_{2}-b_{1}\right)\left(\phi_{b_{2}}-\phi_{b_{1}}\right)} \int_{a_{1}}^{a_{2}} \int_{\phi_{a_{1}}}^{\phi_{a_{2}}} r^{2} J_{m}\left(\alpha_{m n}^{\prime} \frac{r}{a}\right) \cos (m \phi) \mathrm{d} \phi \mathrm{d} r \\
& \times \int_{b_{1}}^{b_{2}} \int_{\phi_{b_{1}}}^{\phi_{b_{2}}} J_{m}\left(\alpha_{m n}^{\prime} \frac{r}{a}\right) \cos (m \phi) \mathrm{d} \phi \mathrm{d} r .
\end{aligned}
$$

If $m>0$,

$$
M_{m n}=\pi a^{2} \frac{\alpha_{m n}^{\prime} J_{m}^{2}\left(\alpha_{m n}^{\prime}\right)-2 m J_{m}\left(\alpha_{m n}^{\prime}\right) J_{m-1}\left(\alpha_{m n}^{\prime}\right)+\alpha_{m n}^{\prime} J_{m-1}^{2}\left(\alpha_{m n}^{\prime}\right)}{2 \alpha_{m n}^{\prime}},
$$

and if $m=0, M_{0 n}=\pi a^{2} J_{0}^{2}\left(\alpha_{0 n}^{\prime}\right) . \rho$ is the density of air, $\delta_{m n}$ is the Kronecker delta, $k_{m n}=\sqrt{\omega^{2} / c^{2}-\alpha_{m n}^{\prime 2} / a^{2}}$ is the wavenumber of the $m n$th mode, $c$ is the speed of sound, $J_{m}$ is the cylindrical Bessel function of the first kind of order $m$, and $\alpha_{m n}^{\prime}$ is the $n$th zero of the derivative of $J_{m}$. In addition, the volume-flow boundary condition was defined such that $\phi_{a_{1}}=-\phi_{a_{2}}$. Each azimuthal evanescent mode will always retain a maximum value in $\phi=0$, since the factor $\left.\cos (m \phi)\right|_{\phi=0}=1$. Due to the spatial extension of the probetube apertures, higher-order evanescent modes are not excited, and a total of 20 modes for $m$ and $n$ were needed for the solution to converge. The inertance was evaluated at frequency $f=1 \mathrm{kHz}$.

\section{B. Results}

In this example, the simulated calibration is carried out in modeled waveguides of lengths $l_{i}=1.4,1.7,2$, and
$2.3 \mathrm{~cm}$, radius $a=4 \mathrm{~mm}$, for comparison with the calibration in the brass waveguides, and inertance $L=37 \mathrm{~kg} / \mathrm{m}^{4}$. The results are calculated for two different simulated evaluation waveguides of the same dimensions as the evaluation waveguides used for the measurements and given in Sec. III B. For the simulated evaluation waveguide (1), $L=37 \mathrm{~kg} / \mathrm{m}^{4}$ and for (2), $L=9 \mathrm{~kg} / \mathrm{m}^{4}$.

Figures 3(a) and 3(b) show the true source impedance given as input to the simulation $Z_{\mathrm{s}}$, the estimated source impedance affected by the parallel compliance $Z_{\mathrm{s}}^{\prime}$, and the parallel coupling $Z_{\mathrm{s}} \| C$ of $Z_{\mathrm{s}}$ and an acoustic compliance $C=37 / Z_{0}^{2}$. It is clear that the two source impedances are not identical and that $Z_{\mathrm{s}}^{\prime}$ is affected by an apparent parallel compliance due to the difference in magnitude and phase shift relative to $Z_{\mathrm{s}}$. This is further indicated by the close resemblance of $Z_{\mathrm{s}}^{\prime}$ with $Z_{\mathrm{s}} \| C$.

Figures 3(c) and 3(d) show impedances and reflectances of the simulated evaluation waveguide (1) using the planewave models $Z_{\mathrm{pw}}$ and $R_{\mathrm{pw}}$, the correct source parameters $Z$ 
(a)

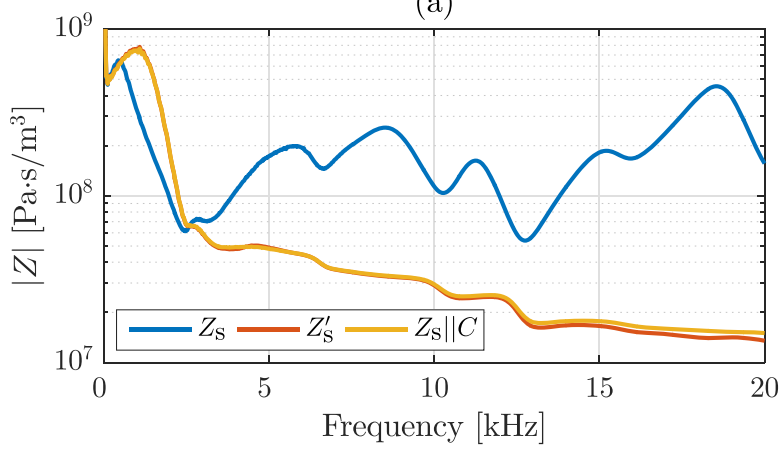

(c)

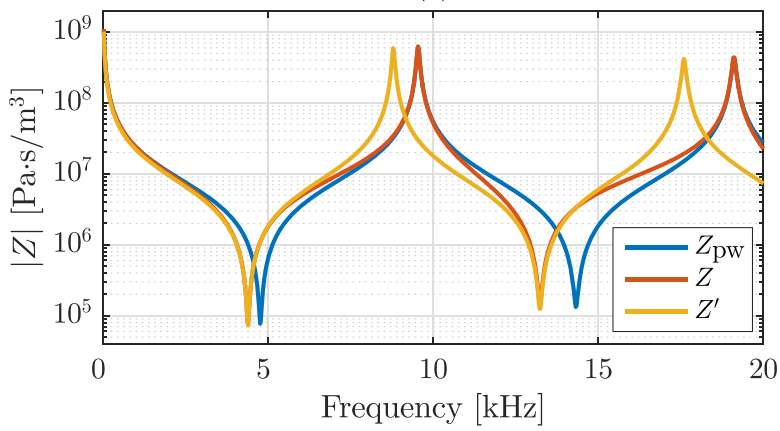

(e)

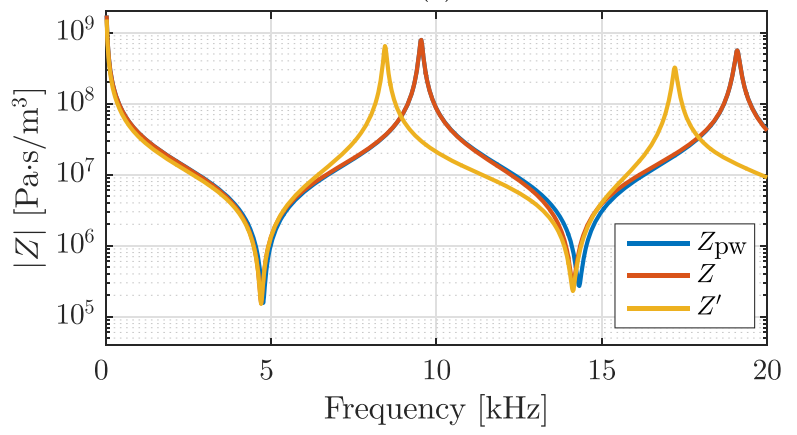

(b)

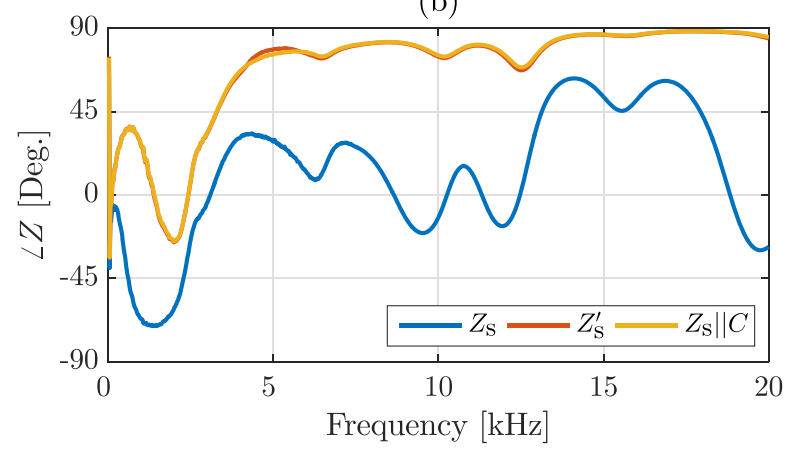

(d)

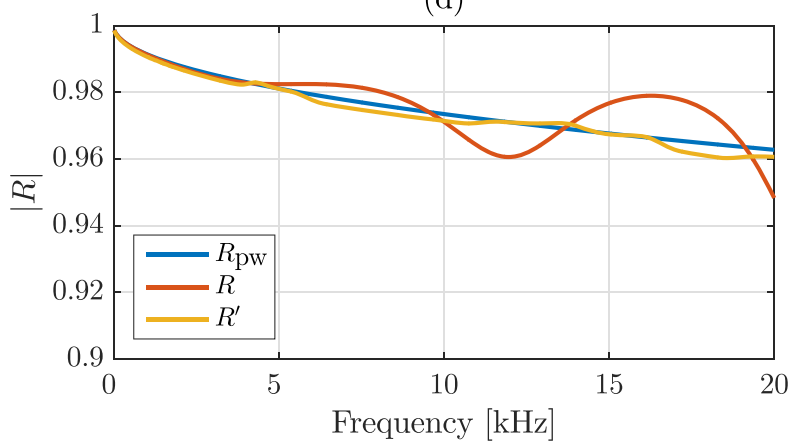

(f)

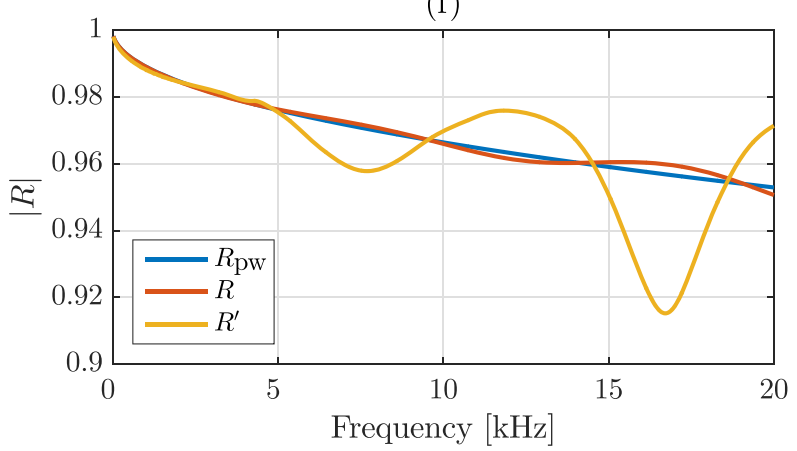

FIG. 3. (Color online) (a),(b) Magnitude and phase of the true source impedance given as input to the simulation $Z_{\mathrm{s}}$, the estimated source impedance affected by the parallel compliance $Z_{\mathrm{s}}^{\prime}$, and the parallel coupling $Z_{\mathrm{s}} \| C$ of $Z_{\mathrm{s}}$ and an acoustic compliance $C=37 / Z_{0}^{2}$. (c),(e) Impedances and (d),(f) reflectances of (c),(d) evaluation waveguide (1), and (e),(f) evaluation waveguide (2). Results are shown using the plane-wave model $Z_{\mathrm{pw}}$ and $R_{\mathrm{pw}}$, the correct source parameters $Z$ and $R$, and the biased source parameters $Z^{\prime}$ and $R^{\prime}$.

and $R$, and the biased source parameters $Z^{\prime}$ and $R^{\prime}$. As hypothesized, $Z^{\prime}$ and $R^{\prime}$ appear to consist of plane waves, corresponding to a seemingly longer waveguide than $Z_{\mathrm{pw}}$ and $R_{\mathrm{pw}}$ due to the positive evanescent-modes inertance. This occurs when impedance minima and maxima are translated equivalently downward in frequency relative to $Z_{\mathrm{pw}}$ and the effects of $C$ and $L$ on $R^{\prime}$, to some degree, cancel each other. Conversely, the proposed calibration methodology is affected exclusively by translated minima in $Z$ relative to $Z_{\mathrm{pw}}$ due to evanescent modes, introducing a discrepancy between $R$ and $R_{\mathrm{pw}}$.

Finally, Figs. 3(e) and 3(f) show $Z_{\mathrm{pw}}, Z, Z^{\prime}, R_{\mathrm{pw}}, R$, and $R^{\prime}$ of the simulated evaluation waveguide (2). Here, the difference in effect of $C$ and $L$ induces large errors into both $Z^{\prime}$ and $R^{\prime}$ as impedance minima and maxima are no longer translated similarly and thus do not cancel. $L$ has been reduced to a low value, minimizing the effect on $Z$ and $R$. The results confirm that, for the considered realization of the existing calibration methodology, evanescent modes introduce an error into the source parameters very similar to that of a parallel acoustic compliance. This introduces errors into impedance measurements in waveguides of radii different from the calibration waveguides.

\section{EXPERIMENTAL RESULTS}

\section{A. Calibration}

To demonstrate the results of the iterative process of determining the factors $L_{i}, L_{i}^{\prime}$, and $\zeta_{i}$ in the proposed calibration methodology, the probe pressures were measured in each of the proposed calibration waveguides and given as input to the calibration program in MATLAB.

Figures 4(a) and 4(b) show the reference impedances $Z_{i}$, estimated impedances $\hat{Z}_{i}$, and relative calibration errors $\epsilon_{i}$ for each calibration waveguide using the physical lengths for calculating the plane-wave impedances $Z_{i, \text { pw }}$ [Eq. (10)]. No further compensation or initial values for $L_{i}, L_{i}^{\prime}$, or $\zeta_{i}$ were applied in this case, i.e., $Z_{i}=Z_{i, \mathrm{pw}}$. The minima of $\hat{Z}_{i}$ are 
(a)

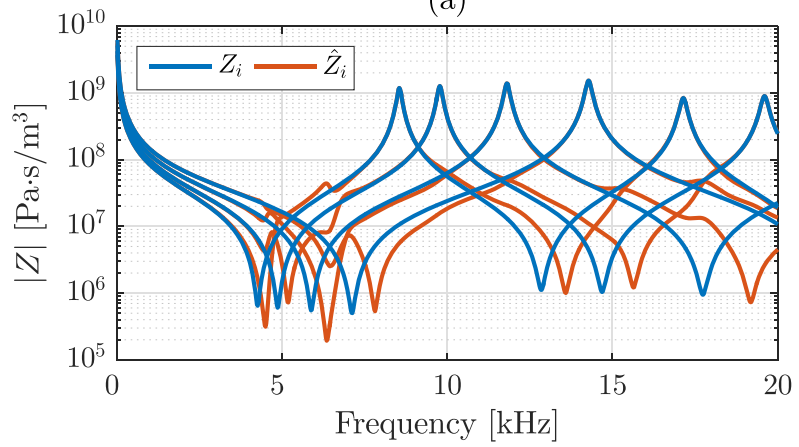

(c)

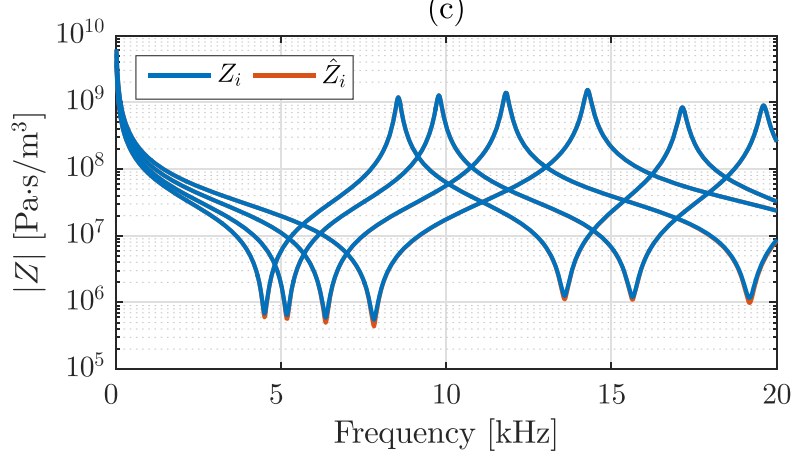

(e)

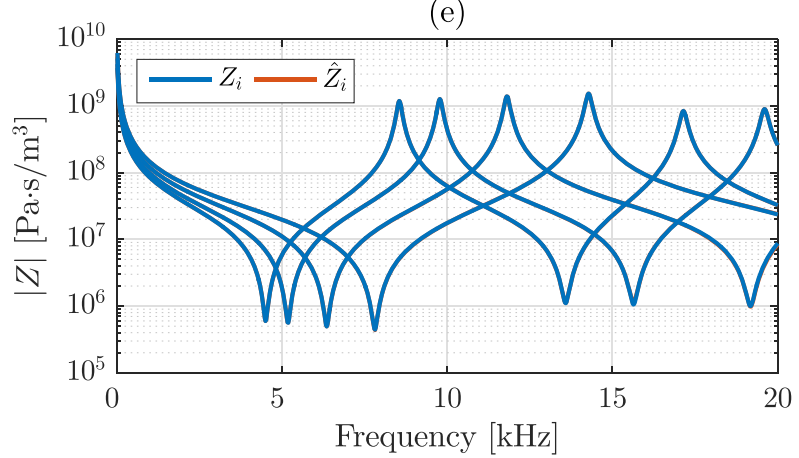

(b)

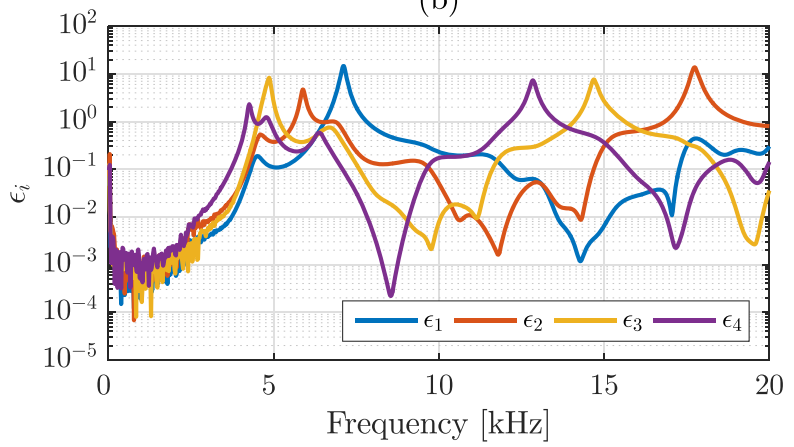

(d)

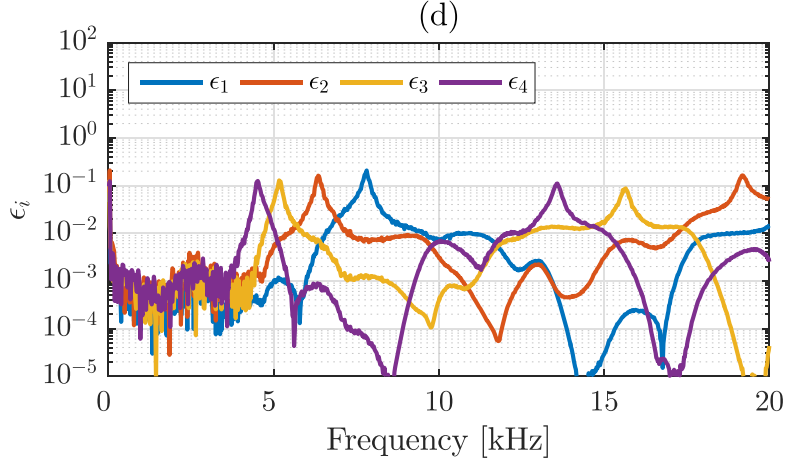

(f)

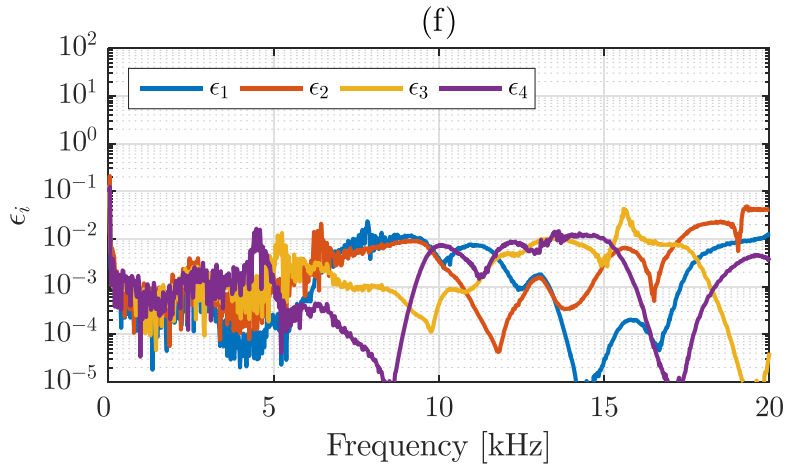

FIG. 4. (Color online) (a),(c),(e) Reference impedances $Z_{i}$, estimated impedances $\hat{Z}_{i}$, and (b),(d),(f) relative calibration errors $\epsilon_{i}$ of the different iterations of the proposed calibration methodology. (a),(b) The result of a calibration, applying the physical lengths of the waveguides without further modification to the plane-wave impedances [Eq. (10) with $Z_{i}=Z_{i, \mathrm{pw}}$ ]. (c),(d) The result of including evanescent modes in $Z_{i}$ [Eq. (14)]. (e),(f) The result of also including the effect of flow losses in $Z_{i}$ [Eq. (15)].

shifted relative to $Z_{i}$ as a result of evanescent modes, resulting in $\epsilon_{i}$ approaching 10 in impedance minima. Also, Figures 4(a) and 4(b) detail how the least-squares cost function [Eq. (2)] weighs the impedance maxima more heavily in calculating the source parameters from the alignment between $Z_{i}$ and $\hat{Z}_{i}$ and the low $\epsilon_{i}$ in impedance maxima.

Figures 4(c) and 4(d) show $Z_{i}, \hat{Z}_{i}$, and $\epsilon_{i}$ as a result of including the evanescent-modes inertances $L_{i}$ in $Z_{i}$. Within the investigated frequency range, two impedance minima are present for the three longer waveguides. Thus, the additional non-linear inertance components $L_{i}^{\prime}$ are included in the model [Eq. (14)]. Figures 4(c) and 4(d) show a significant reduction in $\epsilon_{i}$ and alignment between $Z_{i}$ and $\hat{Z}_{i}$ around impedance minima, while a mismatch in damping still seems to affect these points.

Finally, Figs. 4(e) and 4(f) show $Z_{i}, \hat{Z}_{i}$, and $\epsilon_{i}$ as a result of also including the resistive factors $\zeta_{i}$ associated with the flow losses in $Z_{i}$ [Eq. (15)]. $Z_{i}$ and $\hat{Z}_{i}$ closely align and $\epsilon_{i}$ has now been reduced to a very low level across the entire frequency range. The small remaining $\epsilon_{i}$ across the spectrum is most likely due to noise and slight mismatches between the maxima in $Z_{i}$ and the true transfer impedances $Z_{i \text {,true }}$ between microphone and speaker tube apertures, caused by mechanical tolerances.

For the calibration presented in Fig. 4, the quantities for $L_{i}$ and $\zeta_{i}$ listed in Table I were obtained. $L_{i}$ are negative due to the small diameter of the calibration waveguides. This causes the dominating, lower-order evanescent modes to be out of phase between the probe speaker and microphone. $\zeta_{i}$

TABLE I. The obtained evanescent-modes inertances $L_{i}$ and flow-loss factors $\zeta_{i}$ for the calibration presented in Fig. 4. Subscripts 1-4 indicate the shortest to longest waveguides.

\begin{tabular}{|c|c|c|c|c|}
\hline$i$ & 1 & 2 & 3 & 4 \\
\hline$L_{i}\left(\mathrm{~kg} / \mathrm{m}^{4}\right)$ & -102.6 & -99.1 & -98.3 & -104.2 \\
\hline$\zeta_{i}\left(\mathrm{~Pa} \mathrm{~s}^{3 / 2} / \mathrm{m}^{3}\right)$ & -482.7 & -486.7 & -466.8 & -506.7 \\
\hline
\end{tabular}


are generally negative due to the inherent negative gradient in particle velocity, going away from the speaker probe-tube aperture. The obtained evanescent-modes inertances might seem large; however, the characteristic impedance is similarly large for the small radius. The similarity in obtained values supports the frequency dependency of the evanescent-modes and flow-loss factors since the impedance minima are placed at different points in frequency. The analytical evanescent-modes inertance for the probe and calibration waveguides, calculated from Eq. (21), yielded $L=-97.6 \mathrm{~kg} / \mathrm{m}^{4}$, which is in good agreement with the obtained $L_{i}$. The values for $L_{i}^{\prime}$ are not reported since the deviation from proportionality was minuscule.

Buick et al. (2011) investigated the non-linear effect of acoustic vortex shedding and jet formation at the end of a waveguide on the radiation impedance in terms of an additional non-linear resistance dependent on the acoustic particle velocity. An investigation was carried out to assess the potential influence of this non-linear effect on the proposed calibration methodology. An additional calibration was carried out using a stimulus level $20 \mathrm{~dB}$ lower than the one utilized for the remaining measurements. Except for small variations due to increased noise in these measurements, essentially the same $L_{i}, L_{i}^{\prime}, \zeta_{i}$, and $\epsilon_{i}$ were obtained. It is thus expected that these non-linear effects do not affect the reported measurements in any significant way.

\section{B. Parallel compliance}

To demonstrate the capability of the proposed calibration methodology and benchmark it against the considered realization of the existing methodology, such a calibration was also carried out in the brass waveguides. The source parameters from this calibration $P_{\mathrm{s}}^{\prime}$ and $Z_{\mathrm{s}}^{\prime}$ and the calibration as obtained in Sec. V A, $P_{\mathrm{s}}$ and $Z_{\mathrm{s}}$ were then compared and used for measuring the impedances $Z$ and $Z^{\prime}$ and reflectances $R$ and $R^{\prime}$ of the two evaluation waveguides. Similar to Sec. IV, quantities from the calibration in the brass waveguides, proposedly affected by a parallel compliance, are labeled with prime superscripts.

Figures 5(a) and 5(b) show the source impedances obtained using the proposed methodology $Z_{\mathrm{s}}$, the calibration in the brass waveguides using the existing methodology $Z_{\mathrm{s}}^{\prime}$, and the parallel coupling $Z_{\mathrm{s}} \| C$ of $Z_{\mathrm{s}}$ and an acoustic compliance $C=37 / Z_{0}^{2}$, similar to the simulation. The effect on $Z_{\mathrm{s}}^{\prime}$ is in good agreement with the simulation results in Figs. 3(a) and 3(b). There is a slight discrepancy between $Z_{\mathrm{s}}^{\prime}$ and $Z_{\mathrm{s}} \| C$, presumably due to not having included the material properties of the ear tip in $Z_{\mathrm{s}}$. Still, the parallel compliance appears to have a significantly more profound effect on $Z_{\mathrm{s}}^{\prime}$ compared to these material properties. The variation between $Z_{\mathrm{s}}$ and $Z_{\mathrm{s}} \| C$ at $5 \mathrm{kHz}$, mainly seen in the phase, could also be the result of a small instability in the calculation of the source parameters for the chosen lengths. A similar effect, though much smaller, can be observed in Fig. 3(b).

Figures 5(c) and 5(d) show impedances and reflectances of evaluation waveguide (1) using the source parameters from the proposed methodology $Z$ and $R$, and from the existing methodology $Z^{\prime}$ and $R^{\prime}$. The insertion of the probe using an ear tip thus also gives an indication of the effect of not having the ear tip included in the proposed calibration. However, plane-wave quantities $Z_{\mathrm{pw}}$ and $R_{\mathrm{pw}}$ are not presented as the exact waveguide length is unknown. It is clear that the main difference between $Z$ and $Z^{\prime}$ consists of a translation in impedance maxima between the two calibration methods. Furthermore, $R$ and $R^{\prime}$ seem to be affected by similar errors, in addition to the effect of evanescent modes on $R$ similar to Fig. 3(d). This could be a result of differences in material properties of the ear tip when the probe is removed and reinserted into a waveguide. The discrepancy between $Z_{\mathrm{s}}^{\prime}$ and $Z_{\mathrm{s}} \| C$ in Figs. 5(a) and 5(b) could therefore also result from variations in ear tip material properties between insertions during calibration rather than representing the actual properties of the ear tip material. These results also indicate that these effects are negligible for the utilized ear tip, compared to the effect of the parallel compliance.

Finally, Figs. 5(e) and 5(f) show $Z, Z^{\prime}, R$, and $R^{\prime}$ of evaluation waveguide (2). Due to the construction similar to the proposed calibration waveguides in Fig. 2, the exact lengths are known and the analytical plane-wave quantities $Z_{\mathrm{pw}}$ and $R_{\mathrm{pw}}$ are thus also shown. The results show a translation of maxima in $Z^{\prime}$ and effect on $R^{\prime}$ very similar to the simulations presented in Figs. 3(e) and 5(f). Furthermore, it is evident how the proposed calibration method estimates the position of maxima in $Z$ quite precisely. The small remaining translation of maxima between $Z$ and $Z_{\mathrm{pw}}$ could be the result of mechanical tolerances and having a larger section of the rubber part exposed to the acoustic domain. Still the results indicate that the parallel compliance in the source parameters has been significantly reduced such that the effect is negligible in measurements.

\section{DISCUSSION}

One of the primary limitations in the multi-tube calibration methodology described in existing literature is the incapability of identifying mismatches in impedance maxima. With the length-estimation procedure, which indirectly determines the waveguide lengths from probe-pressure minima that are affected by evanescent modes, and the given error weighting in the least-squares cost function, the source parameters simply align to provide a low calibration error in impedance maxima. This effect is inherently similar to that of an acoustic compliance in parallel with the load and is inherited into the source parameters. The sign of operation of the evanescent-modes inertance $L$ directly affects the sign of operation of the parallel compliance. For positive $L$, impedance minima are translated downward in frequency, whereby a similar translation of maxima in the same direction is achieved, corresponding to a positive compliance $C$ in parallel with the load. However, the sign of operation of the compliance is changed when it is inherited into the source parameters. Measurements are consequently both affected by the parallel compliance and evanescent modes, and for waveguides of radii identical to the calibration waveguides, this causes the measured impedance to mimic a plane-wave impedance. In waveguides of radii different from the calibration waveguides, the contribution from evanescent modes 
(a)

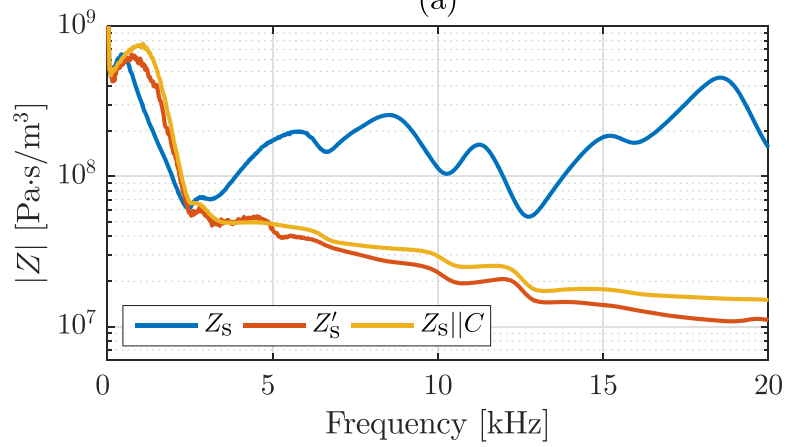

(c)

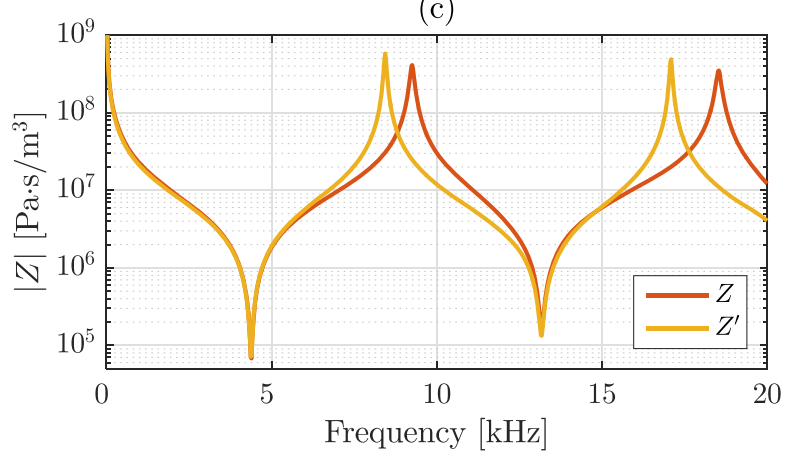

(e)

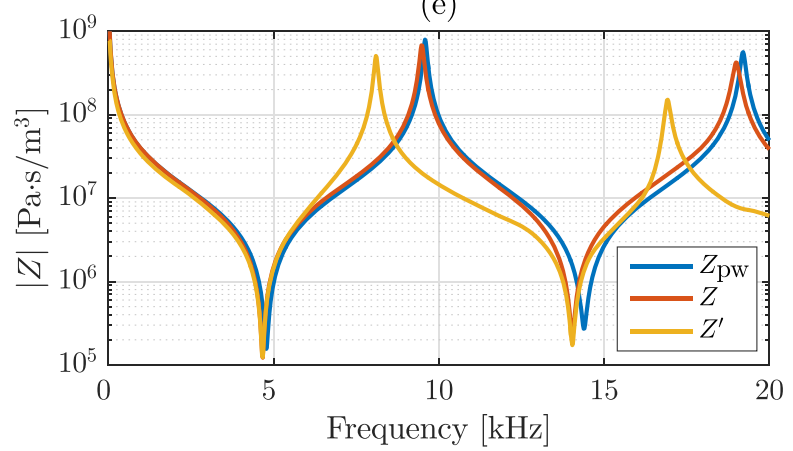

(b)

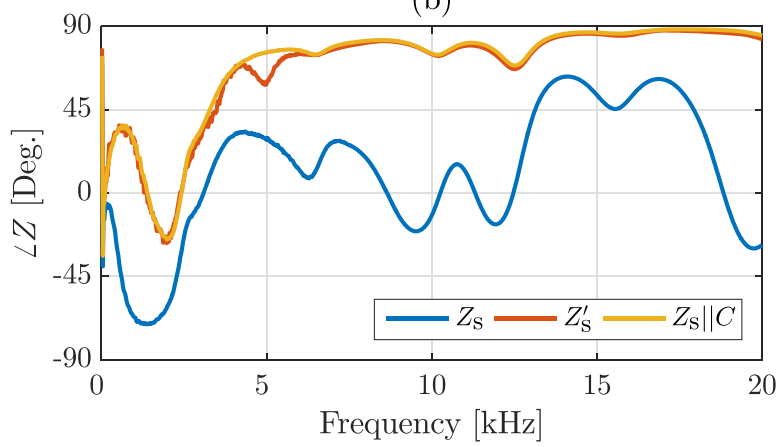

(d)

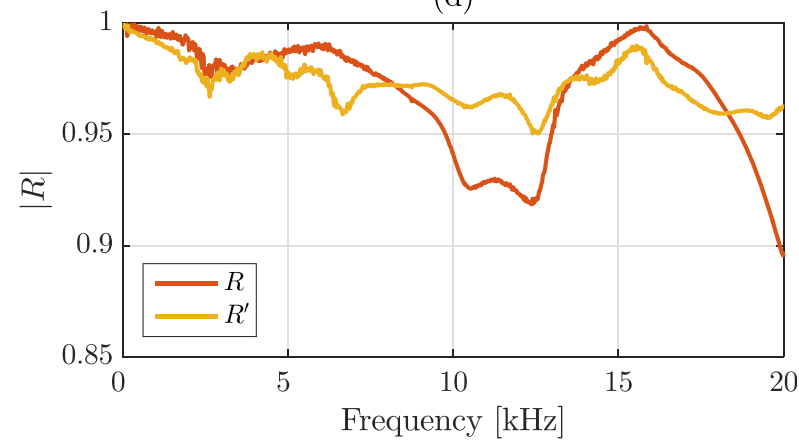

(f)

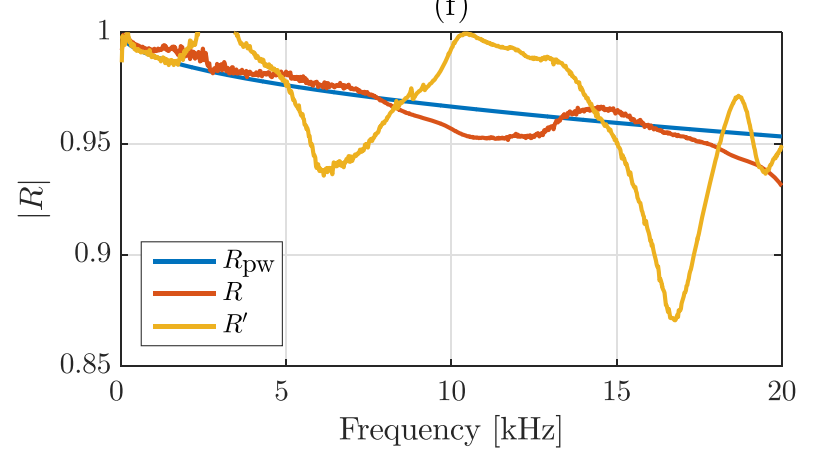

FIG. 5. (Color online) (a),(b) Magnitude and phase of the source impedances obtained using the proposed methodology $Z_{\mathrm{s}}$, the calibration in the brass waveguides using the existing methodology $Z_{\mathrm{s}}^{\prime}$, and the parallel coupling $Z_{\mathrm{s}} \| C$ of $Z_{\mathrm{s}}$ and an acoustic compliance $C=37 / Z_{0}^{2}$. (c),(e) Impedances and (d),(f) reflectances of (c),(d) evaluation waveguide (1), and (e),(f) evaluation waveguide (2). Results are shown using the plane-wave model $Z_{\mathrm{pw}}$ and $R_{\mathrm{pw}}$, the source parameters from the proposed methodology $Z$ and $R$, and from the existing methodology $Z^{\prime}$ and $R^{\prime}$.

changes and the parallel compliance affects the measured impedance differently due to the different characteristic impedance, introducing errors into the measurement. The proposed calibration methodology eliminates the parallel compliance from the source parameters, such that measurements are solely affected by evanescent modes. The method could therefore provide a step forward in increasing the validity and repeatability of ear-canal impedance and reflectance measurements. With the proposed calibration methodology, it is possible to apply the approach of Fletcher et al. (2005) to compensate for the effect of evanescent modes in loads of known radius. However, further research is required as to how these effects can be accounted for in ear canal measurements, where the radius is not easily measured and also varies with probe-insertion depth.

In general, it can be difficult to identify such seemingly parallel components in the probe pressure due to the difference in effect of parallel and series components to the planewave impedance on the calibration error. Any parallel component consistently affecting the probe pressures in each waveguide will simply be inherited into the source parameters and is thus not identifiable in the calibration error. Conversely, series components do affect the error, as is evident from the investigations in this study. This is another reason why the incorrect placement of impedance maxima is not apparent from the calibration error, due to the resemblance with a parallel compliance. In principle, the absolute positioning of impedance maxima using the physical lengths of the waveguides cannot be assessed for the calibration method proposed in this study. Consequently, the methodology is dependent on the mechanical tolerances of the waveguides and supplying correct values for the temperature and atmospheric pressure for calculating the plane-wave impedances of the waveguides. Further research is required as to more thoroughly identify such parallel components in the source parameters and provide an absolute metric for the accuracy and validity of an acoustic Thévenin calibration procedure. 
An argument for inserting the probe into the waveguides using an ear tip during calibration, as practiced in the existing methodology, is that the material properties of the ear tip are included in the source parameters. Voss et al. (2013) demonstrated that the difference in compression of foam ear tips between each insertion in an ear canal is a source of variability in ear-canal reflectance measurements. The results of this paper indicate that such variation likely also affects the material properties of rubber ear tips, though this has not been further investigated. Also, Scheperle et al. (2011) observed a substantial difference in the source impedance, dependent on the utilized ear tip during calibration, i.e., foam versus rubber. The ear tip eliminates the possibility of achieving a well-defined waveguide length during calibration and, in addition, ear tips of many different sizes than the one used during calibration exist. Only a small range of the available rubber ear tips fit into a calibration waveguide of a certain radius. This characterization of the ear-tip material properties within the source parameters might no longer be valid if an ear tip of different size or type is used for the measurement. The results of this study point toward the material properties of rubber ear tips being negligible, at least for the specific, utilized ear tip. However, this might not be the case for ear tips of different sizes of types. If the ear tip is not included in the source parameters, its impedance appears as a parallel component to a measured impedance. It could therefore be extracted from the measurement if the impedance characteristics of the specific ear tip were known and remain unchanged between insertions. It is speculated that the well-defined physical dimensions of the calibration waveguides, facilitated by the removal of the rubber ear tip, outweigh the possible advantages of having it included in the calibration. However, more research is required to more thoroughly assess this effect of the ear tip on the source parameters in the absence of any parallel compliance inherited from the calibration procedure.

The actual physical relationship with frequency of the additional term to account for non-proportionality in evanescent modes in Eq. (14) is only crucial when two or more impedance minima are present within the frequency range. The term merely serves to model the slight difference in translation between two impedance minima that cannot be modeled by an ideal inertance. A more accurate form would require additional terms accounting for the contribution of each evanescent mode. However, it is only valid for the assumed case that the probe is positioned exactly in the input plane. Small offsets of the probe relative to the input plane due to mechanical tolerances will induce a fundamentally different relationship. If longer waveguides are used for the calibration or several impedance minima are present, it might be necessary to include some of these additional factors to correctly align impedance minima and achieve a low calibration error. Additionally, the formulation in Eq. (21) assumes a uniformly injected velocity profile from the speaker tube. This tube typically has a hydraulic diameter comparable to the acoustic velocity boundary layer and, consequently, this dependency with frequency on injected velocity profile will introduce an additional uncertainty into the evanescent modes. The discrepancy between reference and estimated impedances due to flow losses are minuscule and are only significant in the calibration since very low magnitudes of impedance are present in impedance minima. Like evanescent modes, these flow losses also affect subsequent measurements in ear canals, but impedance minima are typically more damped in this case due to the resistive behavior of the tympanic membrane. In the utilized probe, the speaker and microphone tubes in the probe tube are separated by a $0.3 \mathrm{~mm}$ wall and the effect of flow losses could be reduced by physically separating them farther apart. However, this would reduce the high-frequency performance and thereby increase the effect of cross talk in the probe on measurements, since the probe tubes would have to be narrowed.

\section{CONCLUSION}

This paper has proposed an alternative method for calibrating an ear probe to determine its Thévenin-equivalent source parameters and facilitate the measurement of acoustic impedance. It has been demonstrated how one possible realization of a widely used, existing calibration methodology indirectly estimates the waveguide model lengths based on probe-pressure minima that are affected by evanescent modes, and how this induces a parallel acoustic compliance into the source parameters. It is likely that other realizations of this existing methodology are also affected by such errors. A simulation was set up to investigate the effect of the acoustic length estimation on the source parameters and measurements in waveguides of radii different from the calibration waveguides, which showed that this can lead to large errors in measurements of impedance and reflectance. Furthermore, the iterative steps comprised by the proposed calibration method were described and validated in terms of a calibration of an actual probe. This demonstrated how the calibration error could be reduced to a very low level across frequency. Finally, the predicted behavior of the acoustic length estimation of the existing calibration methodology was confirmed from a translation of the impedance maxima in the measured impedance in a waveguide of radius different from the calibration waveguides. In this case, the proposed calibration method yielded the correct plane-wave impedance with aligned impedance maxima, though still affected by evanescent modes.

\section{ACKNOWLEDGMENTS}

The authors would like to thank Stephen T. Neely for helpful discussions and the two anonymous reviewers for comments and suggestions for the manuscript. This research was funded by Interacoustics $\mathrm{A} / \mathrm{S}$ and the Innovation Fund Denmark under File No. 5189-00100B.

\footnotetext{
${ }^{1}$ www.playrec.co.uk (Last viewed November 3, 2017).

${ }^{2}$ Initially, the built-in ER-10X (Etymōtic Research, Inc., Elk Grove Village, IL) calibration waveguide of adjustable length was used. However, a small leak in the piston termination of this waveguide rendered the distinction between effects arising from the ear tip and the finite termination impedance difficult. If not properly accounted for, errors due to the leak were accordingly introduced into measurements below $1 \mathrm{kHz}$.
} 
Allen, J. B. (1985). "Measurement of eardrum acoustic impedance," in Peripheral Auditory Mechanisms (Springer-Verlag, New York), pp. 44-51.

Buick, J. M., Atig, M., Skulina, D. J., Campbell, D. M., Dalmont, J. P., and Gilbert, J. (2011). "Investigation of non-linear acoustic losses at the open end of a tube," J. Acoust. Soc. Am. 129, 1261-1272.

Charaziak, K. K., and Shera, C. A. (2017). "Compensating for ear-canal acoustics when measuring otoacoustic emissions," J. Acoust. Soc. Am. 141, 515-531.

Ellison, J. C., Gorga, M., Cohn, E., Fitzpatrick, D., Sanford, C. A., and Keefe, D. H. (2012). "Wideband acoustic transfer functions predict middle-ear effusion," Laryngoscope 122, 887-894.

Feeney, M. P., and Keefe, D. H. (2001). "Estimating the acoustic reflex threshold from wideband measures of reflectance, admittance, and power," Ear Hear. 22, 316-332.

Fletcher, N. H., Smith, J., Tarnopolsky, A. Z., and Wolfe, J. (2005). "Acoustic impedance measurements-Correction for probe geometry mismatch," J. Acoust. Soc. Am. 117, 2889-2895.

Huang, G. T., Rosowski, J. J., Puria, S., and Peake, W. T. (2000). "A noninvasive method for estimating acoustic admittance at the tympanic membrane," J. Acoust. Soc. Am. 108, 1128-1146.

Keefe, D. H. (1984). "Acoustical wave propagation in cylindrical ducts: Transmission line parameter approximations for isothermal and nonisothermal boundary conditions," J. Acoust. Soc. Am. 75, 58-62.

Keefe, D. H., and Benade, A. H. (1981). "Impedance measurement source and microphone proximity effects," J. Acoust. Soc. Am. 69, 1489-1495.

Keefe, D. H., Folsom, R. C., Gorga, M. P., Vohr, B. R., Bulen, J. C., and Norton, S. J. (2000). "Identification of neonatal hearing impairment: Earcanal measurements of acoustic admittance and reflectance in neonates," Ear Hear. 21, 443-461.

Keefe, D. H., Ling, R., and Bulen, J. C. (1992). "Method to measure acoustic impedance and reflection coefficient," J. Acoust. Soc. Am. 91, 470-485.

Keefe, D. H., Sanford, C. A., Ellison, J. C., Fitzpatrick, D. F., and Gorga, M. P. (2012). "Wideband aural acoustic absorbance predicts conductive hearing loss in children,” Int. J. Audiol. 51, 880-891.
Lewis, J. D., McCreery, R. W., Neely, S. T., and Stelmachowicz, P. G. (2009). "Comparison of in-situ calibration methods for quantifying input to the middle ear," J. Acoust. Soc. Am. 126, 3114-3124.

McCreery, R. W., Pittman, A., Lewis, J., Neely, S. T., and Stelmachowicz, P. G. (2009). "Use of forward pressure level to minimize the influence of acoustic standing waves during probe-microphone hearing-aid verification," J. Acoust. Soc. Am. 126, 15-24.

Merchant, G. R., Röösli, C., Niesten, M. E. F., Hamade, M. A., Lee, D. J., McKinnon, M. L., Ulku, C. H., Rosowski, J. J., Merchant, S. N., and Nakajima, H. H. (2014). "Power reflectance as a screening tool for the diagnosis of superior semicircular canal dehiscence," Otol. Neurotol. 36, 172-177.

Piskorski, P., Keefe, D. H., Simmons, J. L., and Gorga, M. P. (1999). "Prediction of conductive hearing loss based on acoustic ear-canal response using a multivariate clinical decision theory," J. Acoust. Soc. Am. 105, 1749-1764.

Scheperle, R. A., Goodman, S. S., and Neely, S. T. (2011). "Further assessment of forward pressure level for in situ calibration," J. Acoust. Soc. Am. 130, 3882-3892.

Scheperle, R. A., Neely, S. T., Kopun, J. G., and Gorga, M. P. (2008). "Influence of in situ, sound-level calibration on distortion-product otoacoustic emission variability," J. Acoust. Soc. Am. 124, 288-300.

Siegel, J. H., and Neely, S. T. (2017). "Eartip modification greatly reduces evanescent waves," in Proc. 40th MidWinter Meet., Association for Research in Otolaryngology.

Souza, N. N., Dhar, S., Neely, S. T., and Siegel, J. H. (2014). "Comparison of nine methods to estimate ear-canal stimulus levels," J. Acoust. Soc. Am. 136, 1768-1787.

Voss, S. E., and Allen, J. B. (1994). "Measurement of acoustic impedance and reflectance in the human ear canal," J. Acoust. Soc. Am. 95, 372-384.

Voss, S. E., Stenfelt, S., Neely, S. T., and Rosowski, J. J. (2013). "Factors that introduce intrasubject variability into ear-canal absorbance measurements," Ear Hear. 34, 60S-64S.

Withnell, R. H., Jeng, P. S., Waldvogel, K., Morgenstein, K., and Allen, J. B. (2009). "An in situ calibration for hearing thresholds," J. Acoust. Soc. Am. 125, 1605-1611. 